\title{
Controlled Morphology of Porous Polyvinyl Butyral Nanofibers
}

\author{
Daniela Lubasova and Lenka Martinova \\ Department of Nonwovens, Technical University of Liberec, 46117 Liberec, Czech Republic \\ Correspondence should be addressed to Daniela Lubasova, daniela.lubasova@tul.cz
}

Received 12 August 2010; Revised 3 December 2010; Accepted 17 January 2011

Academic Editor: Theodore Tsotsis

Copyright ( 2011 D. Lubasova and L. Martinova. This is an open access article distributed under the Creative Commons Attribution License, which permits unrestricted use, distribution, and reproduction in any medium, provided the original work is properly cited.

\begin{abstract}
A simple and effective method for the fabrication of porous nanofibers based on the solvent evaporation methods in one-step electrospinning process from the commercial polyvinyl butyral (PVB) is presented. The obtained nanofibers are prevalently amorphous with diameters ranging from 150 to $4350 \mathrm{~nm}$ and specific surface area of approximately $2-20 \mathrm{~m}^{2} / \mathrm{g}$. Pore size with irregular shape of the porous PVB fibers ranged approximately from 50 to $200 \mathrm{~nm}$. The effects of polymer solution concentration, composition of the solvents mixture, and applied voltage on fiber diameter and morphology were investigated. The theoretical approach for the choice of poor and good solvents for PVB was explained by the application Hansen solubility parameter (HSP) and two-dimensional graph. Three basic conditions for the production of porous PVB nanofibers were defined: (i) application of good/poor solvent mixture for spinning solution, (ii) differences of the evaporation rate between good/poor solvent, and (iii) correct ratios of good/poor solvent (v/v). The diameter of prepared porous PVB fibers decreased as the polymer concentration was lowered and with higher applied voltage. These nanofiber sheets with porous PVB fibers could be a good candidate for highefficiency filter materials in comparison to smooth fibers without pores.
\end{abstract}

\section{Introduction}

Many literature references discuss the production of porous nanofibers in the electrospinning process. There are a lot of methods, such as the extraction of a component from bicomponent nanofibers, phase separation during electrospinning, and production of porous nanofibers under specific process conditions (temperature, humidity). These methods are technically difficult and demand two step production or special additional device [1-3].

First idea for producing the porous nanofibers in one step arose after studying works published by Elford [4] and Ferry [5]. One of the earliest methods of making microporous membranes was described there. In the method's simplets form, a polymer is dissolved in a two-component solvent mixture consisting of volatile good solvent and an involatile poor solvent for a polymer. This two-component polymer solution is cast on a glass plate. As the good, volatile solvent evaporates the casting solution is enriched in the poor, non-volatile solvent. The polymer precipitates, forming the porous membrane structure.
An interesting finding is that the use of evaporation of solvents yields polymer fibers with a relatively regular porous structure. The regular phase morphology is induced by rapid phase separation in an electrospinning jet when a highly volatile solvent is used. The solvent-rich regions in the jet result in the pores after its intensive evaporation.

The development of porous fibers by electrospinning was studied by Lubasova and Martinova [6], where porous PCL nanofibers from a nonsolvent/solvent/polymer system were prepared. The same principle is described in the reference of Qi et al. [7], where an alcohol was used as a non-solvent and dichloromethane as a solvent for PLA. It was confirmed that a porous structure of nanofibers could be obtained by varying the ratio of non-solvent/solvent in the ternary system with different values of evaporation rate.

It was proved that porous nanofibers are more efficient for cultivation of different cells and the growth could be influenced by the morphology of the nanofibers in previous work. The cells attach easily to porous nanofibers and show better growth than the cells grown on nonporous nanofibers. Khil et al. [8] prepared a porous polycaprolactone (PCL) 


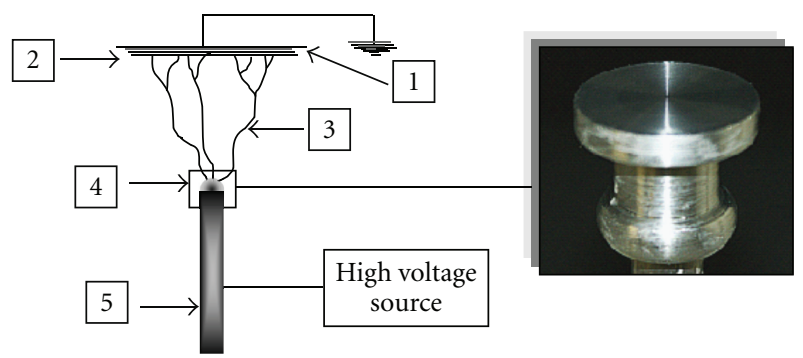

FIGURE 1: Schematic of a simple electrospinning experiment; (1) electrode grounding shield, (2) nanofiber sheet, (3) direction of nanofiber formation, (4) a special flat electrode with polymer solution, and (5) a positive electrode.

filament by an electrospinning process and studied the growth characteristics of cells on these nonwoven fabrics. This work revived the important role of scaffold porosity for proliferation of living animal cells.

\section{Materials and Method}

PVB (Mowital $\mathrm{B} 60 \mathrm{H}$, dynamic viscosity of $10 \%$ solution in ethanol 160-260 $\mathrm{mPa} \cdot \mathrm{S}$ ) was purchased from Kuraray. Several spinning solutions were prepared by dissolving PVB in the different types and quantities of solvents and their mixtures (methanol, ethanol, tetrahydrofuran (THF), dimethylsulfoxide (DMSO)). The total polymer concentration was fixed at 8 and $10 \mathrm{wt} \%$. PVB solutions in solvent mixtures were prepared by magnetic stirring at room temperature overnight.

A schematic representation of the equipment used in the laboratory for electrospinning is depicted in Figure 1. The electrospinning process of PVB solutions was carried out at a voltage of 30 or $35 \mathrm{kV}$ and the electrode-to-collector distance was fixed at $10 \mathrm{~cm}$.

\section{Experimental Results and Discussion}

3.1. Theoretical Approach for Choice of Optimal Solvents. HSP was used for the prediction of the solubility of polymers in various solvents or their mixture. The application of a solubility parameter is the practical way of predicting the polymer solubility or quality of the solvent. Hansen [9] derived a practical extension of Hildebrand solubility parameter into three parts: polar force component $\left(\delta_{\mathrm{p}}\right)$, dispersion force component, $\left(\delta_{\mathrm{d}}\right)$ and hydrogen bonding component $\left(\delta_{\mathrm{h}}\right)$ :

$$
\delta_{\text {total }}^{2}=\delta_{\mathrm{d}}^{2}+\delta_{\mathrm{p}}^{2}+\delta_{\mathrm{h}}^{2} .
$$

HSP for polymers is commonly obtained by an indirect method which involves testing the relevant material in contact with many solvents under conditions relevant to a given problem. When the bond energy of the material is similar to that of a given solvent, it will dissolve, swell, or adsorb onto the material. An accurate prediction of solubility parameter components from the chemical structure is difficult because the interaction of different structural groups

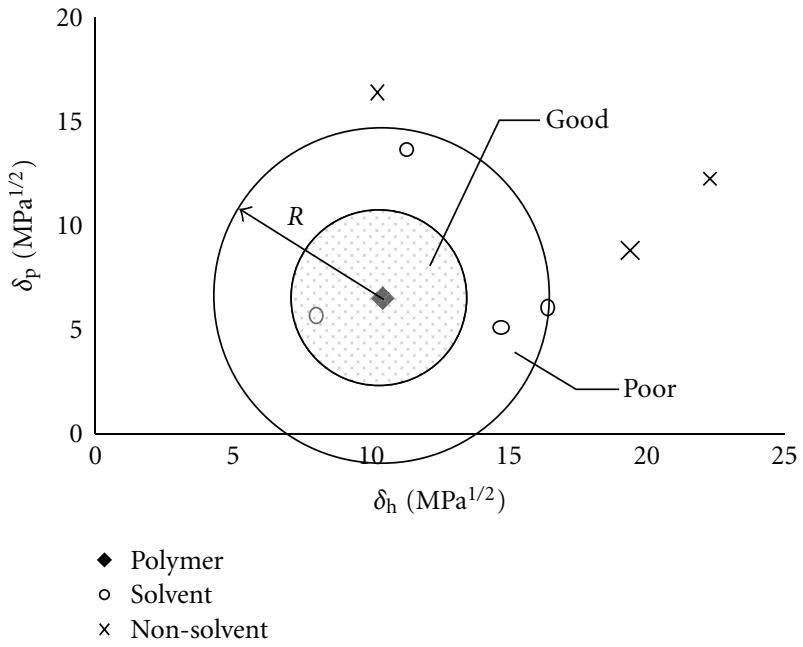

FIGURE 2: The solubility sphere for a polymer.

producing overall polar and hydrogen-bonding properties does not obey simple rules. Despite this, a useful prediction method based on molecular structure has been proposed by Van Krevelen [10]. According to Van Krevelen each parameter can be estimated using (2), where $F_{\mathrm{d}}$ is the dispersion component, $F_{\mathrm{p}}$ the polar component, $E_{\mathrm{h}}$ the contribution of hydrogen bond forces to the cohesive energy, $i$ the contributing group, and $V_{m}$ the molar volume of the polymer:

$$
\delta_{\mathrm{d}}=\frac{\sum F_{\mathrm{d} i}}{V_{m}}, \quad \delta_{\mathrm{p}}=\frac{\sqrt{\sum F_{\mathrm{p} i}^{2}}}{V_{m}}, \quad \delta_{\mathrm{h}}=\sqrt{\frac{\sum E_{\mathrm{h} i}}{V_{m}}} .
$$

Hansen used a three-dimensional geometrical model to the interpret solubility of a polymer by a graphical method. Hansen's three-dimensional volume can be similarly illustrated in a two-dimensional (2D) graph that uses only two of the three parameters, most commonly $\delta_{\mathrm{p}}$ and $\delta_{\mathrm{h}}$, see Figure 2. This model introduces a "solubility sphere" of a polymer as a circle in a $2 \mathrm{D}$ system where the axes are represented by the two solubility components $\left(\delta_{\mathrm{p}}\right.$ and $\left.\delta_{\mathrm{h}}\right)$. The centre of the solubility sphere is located at the point $\left({ }^{\mathrm{P}} \delta_{\mathrm{p}},{ }^{\mathrm{P}} \delta_{\mathrm{h}}\right)$ with HSP of the polymer. The radius of the solubility sphere is the interaction radius $R$. Solvent points are represented by HSP of solvents $\left({ }^{S} \delta_{\mathrm{p}},{ }^{\mathrm{S}} \delta_{\mathrm{h}}\right)$. If a solvent point is located at the centre of the solubility sphere, then the polymer can be dissolved by the solvent. On the other hand, if the solvent point is placed outside the solubility sphere, such solvent does not dissolve the polymer.

3.2. Preparation and Spinning of PVB Solutions. Determination of the good and poor solvents for PVB and processing of porous nanofibers was the main goal. The solubility parameter of the polymer PVB was predicted using (2) to calculate $\delta_{\mathrm{d}}, \delta_{\mathrm{p}}$, and $\delta_{\mathrm{h}}$ which are shown in Table 1 . For a more detailed explanation refer to Van Krevelen [10].

The $2 \mathrm{D}$ graph was constructed in the first step. This graph uses only two of the three HSP components, particularly $\delta_{\mathrm{p}}$ and $\delta_{\mathrm{h}}$; see Figure 3 . 
TABLe 1: HSP calculation for PVB using the Hoftyzer-Van Krevelen method.

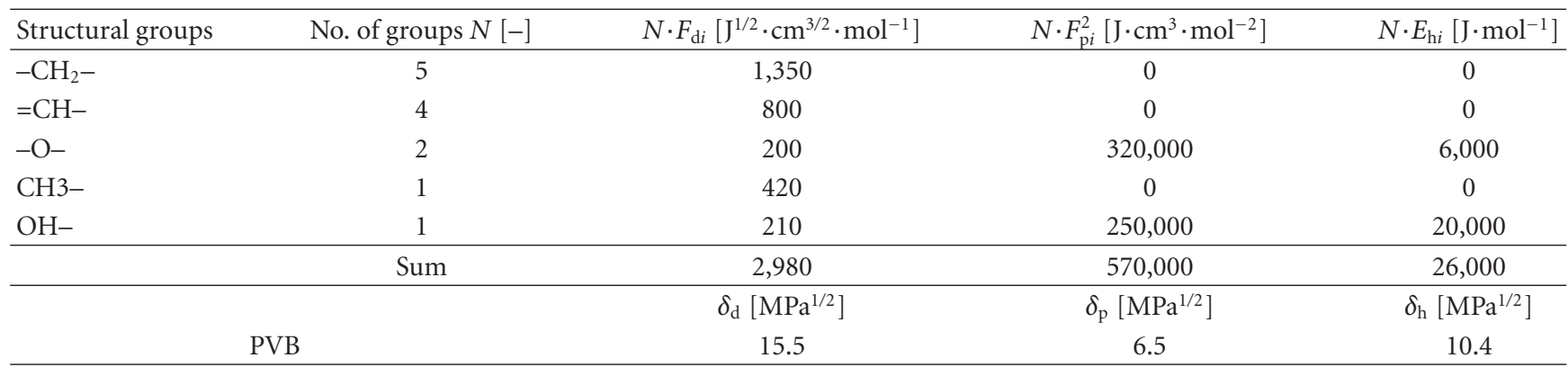

TABLE 2: Quality of solvents for dissolving PVB.

\begin{tabular}{lccc}
\hline Polymer/solvents & \multicolumn{1}{c}{ PVB } & Vapor pressure $\left(25^{\circ} \mathrm{C}\right)[\mathrm{kPa}]$ \\
\hline THF & 2.5 & Character of the solvent & 26.27 \\
DMSO & 9.9 & Good & 0.08 \\
Ethanol & 9.3 & Poor & 24.60 \\
Methanol & 13.2 & Poor & 32.7 \\
\hline
\end{tabular}

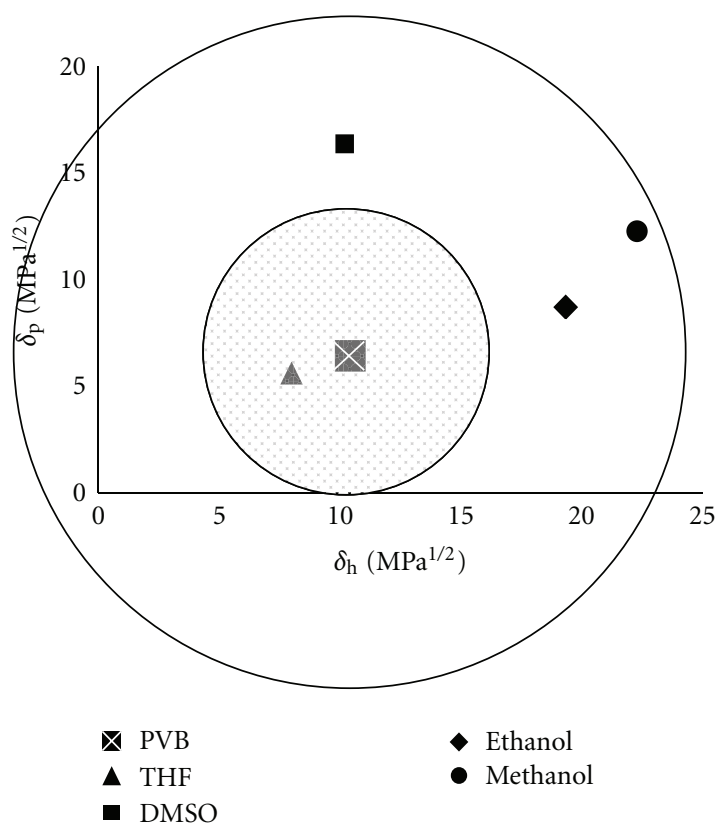

Figure 3: Two-dimensional graph for prediction of good or poor solvent for PVB: grey area implies poor solvent; white area implies good solvent.

TABLE 3: Solvent mixtures used as PVB solvents for electrospinning.

Solvent mixtures
Ethanol/methanol $(9 / 1,8 / 2$ and $7 / 3 \mathrm{v} / \mathrm{v})$
Ethanol/DMSO $(9 / 1,8 / 2$ and $7 / 3 \mathrm{v} / \mathrm{v})$
THF/DMSO $(95 / 5,9 / 1$ and $8 / 2 \mathrm{v} / \mathrm{v})$

Secondly, a parameter $\Delta \delta_{(\mathrm{S}-\mathrm{P}) 2 \mathrm{D}}$ was evaluated, which is given by the distance between the HSP of solvent-point and
TABLE 4: Morphology of PVB fibers.

\begin{tabular}{ll}
\hline Solvent mixtures & Morphology of nanofibers \\
\hline Ethanol/methanol 9/1 (v/v) & nonporous \\
Ethanol/DMSO 9/1 (v/v) & partly porous \\
THF/DMSO 95/5 (v/v) & porous \\
THF/DMSO 9/1 (v/v) & porous \\
\hline
\end{tabular}

the centre of the solubility sphere in the 2D graph, see (3). This parameter can be simply used for prediction of the poor or good solvent. It is expected that the low values of this parameter signify good solvent while higher values indicate poor solvent (see Table 2):

$$
\Delta \delta_{(\mathrm{S}-\mathrm{P}) 2 \mathrm{D}}=\left[\left({ }^{\mathrm{S}} \delta_{\mathrm{p}}-{ }^{\mathrm{P}} \delta_{\mathrm{p}}\right)^{2}+\left({ }^{\mathrm{S}} \delta_{\mathrm{h}}-{ }^{\mathrm{P}} \delta_{\mathrm{h}}\right)^{2}\right]^{1 / 2}
$$

Several spinning solutions were prepared by dissolving PVB in a mixture of different good and poor solvent combinations (ethanol/methanol, ethanol/DMSO and THF/DMSO). The volume ratios of good and poor solvents were varied; see Table 3. The concentration of polymer solutions was a constant $10 \mathrm{wt} . \%$.

3.3. The Effect of the Mixture of Good/Poor Solvents on Fiber Morphology. The nanofiber sheets were observed by a scanning electron microscope (SEM). It was found that electrospinning of PVB led to porous, nonporous, or partly porous fibers; see Table 4. The electrospinning process was intensive in all cases. Figure 4 shows SEM images of the PVB fibers with the effect of the mixture of good and poor solvents on the fiber morphology.

Examination of the SEM images (Figure 4) indicates that the PVB fibers obtained from the solvent mixture THF/DMSO $(9 / 1 \mathrm{v} / \mathrm{v})$ exhibit a highly porous structure with 


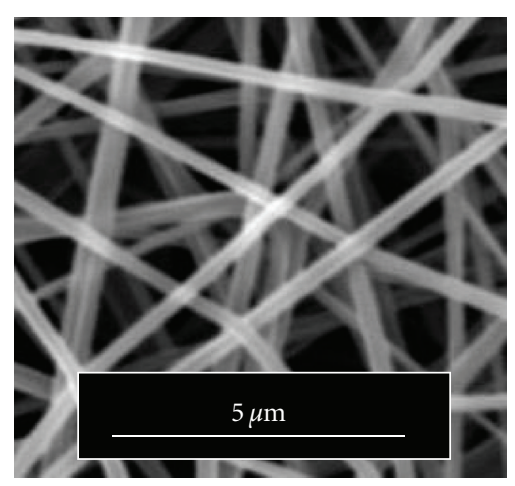

(a)

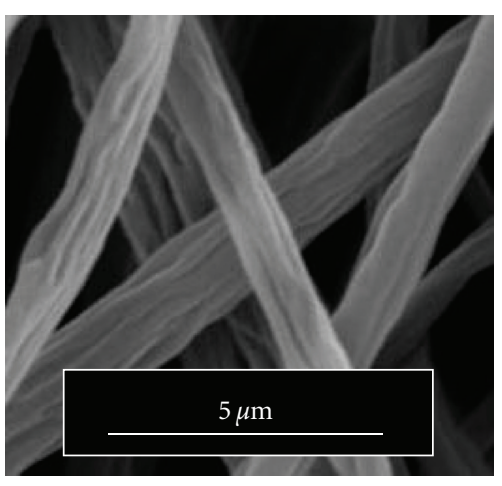

(b)

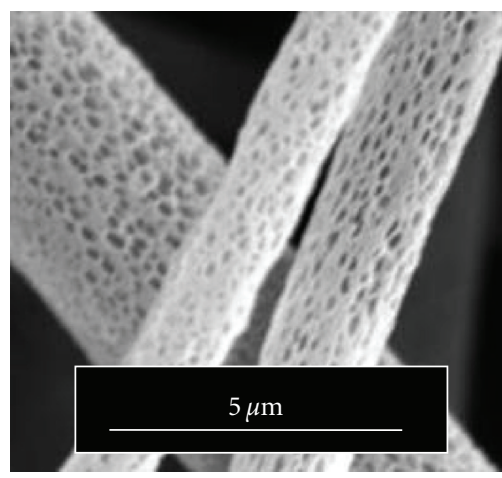

(c)

FIGURE 4: SEM images of PVB nanofibers prepared from $10 \mathrm{wt} . \%$ polymer solution from the mixtures: (a) ethanol/methanol ( $9 / 1 \mathrm{v} / \mathrm{v}),(\mathrm{b})$ ethanol/DMSO (9/1 v/v), (c) THF/DMSO (9/1 v/v).

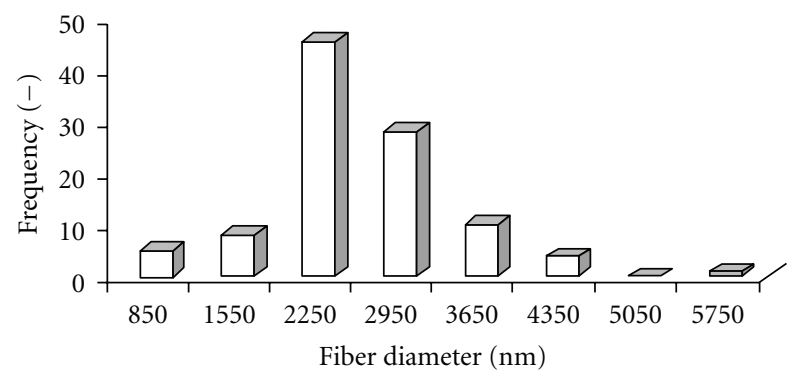

FIGURE 5: Histogram of the fiber diameter for electrospun PVB $10 \mathrm{wt} . \%$ solution from the electrode-to-collector distance of $10 \mathrm{~cm}$ and applied voltage of $30 \mathrm{kV}$.

a pore size of approximately $100 \mathrm{~nm}$. The elliptically shaped pores covered the entire surface of the fibers. Electrospinning of PVB from ethanol/DMSO 9/1 (v/v) led to partially porous fibers with smaller fiber diameters. Non-porous nanofibers were prepared from the mixture of the ethanol/methanol 9/1 (v/v). The SEM images shown in Figure 4 imply that the emerging porous structure of fibers is observed only for the mixtures of good and poor solvents with the higher difference in the evaporation rate between good and poor solvents. The results in Table 2 show the smallest value of vapor pressure for DMSO in comparison with other solvents.

A difference in porous and partially porous nanofibers may be attributed to the parameter $\Delta \delta_{(\mathrm{S}-\mathrm{P}) 2 \mathrm{D}}$. In the case of partially porous fibers, the values of $\Delta \delta_{\text {(S-P) } 2 \mathrm{D}}$ parameter for ethanol and DMSO are 9.3 and $9.9 \mathrm{MPa}^{1 / 2}$, respectively. In comparison with porous fibers, the values of parameter $\Delta \delta_{(\mathrm{S}-\mathrm{P}) 2 \mathrm{D}}$ for THF and DMSO are 2.5 and $9.9 \mathrm{MPa}^{1 / 2}$, respectively. These differences play a significant role for the production of porous fibers.

The described method for the preparation of porous fibers was successful although it leads to higher fiber diameters; see Figure 5. Histograms of fiber diameters were obtained from the image analysis LUCIA G. The average fiber diameter of these fibers was $2546 \mathrm{~nm}$. For that reason, decreasing the fiber diameter by the effect of lower concentration or higher applied voltage during process was solved.
3.4. The Effect of PVB Concentration on Fiber Diameter. The electrospinning of $8 \mathrm{wt} . \%$ solutions PVB prepared from the mixture of the THF/DMSO and the effect of the ratio of good and poor solvent were investigated. The pore size of electrospun nanofibers formed by the good and poor solvent evaporation method is influenced by many factors. In general, increasing the ratio of the poor solvent or decreasing the polymer concentration changes the porosity. The SEM images confirm that the quantity of the pores on the fibers increases when the ratio of good and poor solvent decreases (Figure 6).

The histogram of the fiber diameters shows the reduction of fiber diameter by decreasing PVB concentration from 10 to $8 \mathrm{wt} . \%$. The average fiber diameter of these fibers was $605 \mathrm{~nm}$ (Figure 7).

3.5. The Effect of Applied Voltage on Fiber Diameter. It was found that the small change of voltage from 30 to $35 \mathrm{kV}$ influences both fiber diameter and porosity. Solutions of PVB in the mixture of the THF/DMSO $(9 / 1 \mathrm{v} / \mathrm{v})$ with a concentration of $10 \mathrm{wt} . \%$ was electrospun. SEM micrographs (Figure 8) show the result. The jet of the polymer fluid can easily start the phase separation with a lower voltage of $30 \mathrm{kV}$, and the structure is more porous. Diffusivity of good and poor solvents reduces the difference of the vapor pressure of the components when increasing the voltage. Therefore nanofibers with lower porosity and smaller fiber diameter are obtained. The average fiber diameter of these fibers was $1411 \mathrm{~nm}$; see Figure 9.

\section{Conclusion}

The results show that the vapor pressure of the solvents plays an important role in fiber diameter and porosity. The dependence of nanofiber morphology on different solvent mixtures (good and poor), volume ratios of good and poor solvent and the concentration of polymer solution were studied. HSP was found as a useful tool for the prediction of suitable solvents for preparation of the porous nanofibers via the electrospinning process. All three defined conditions for 


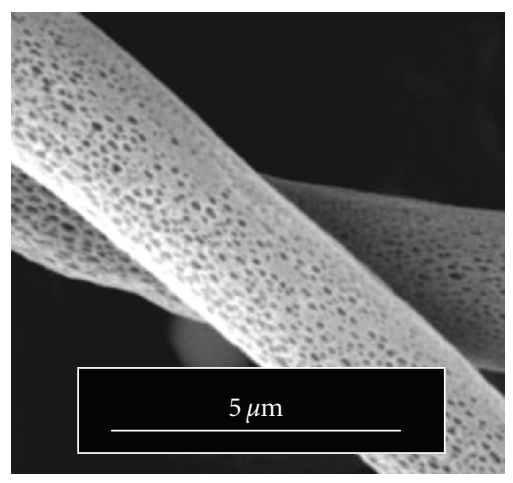

(a)

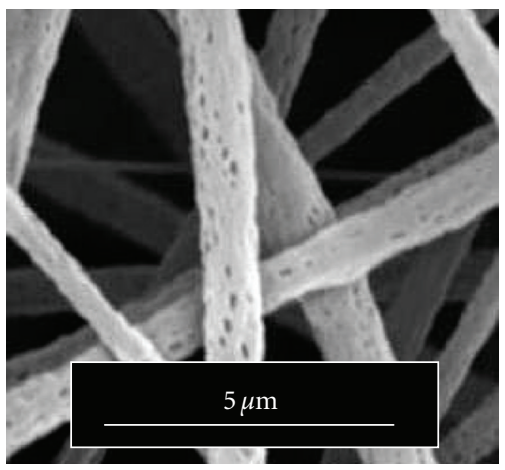

(c)

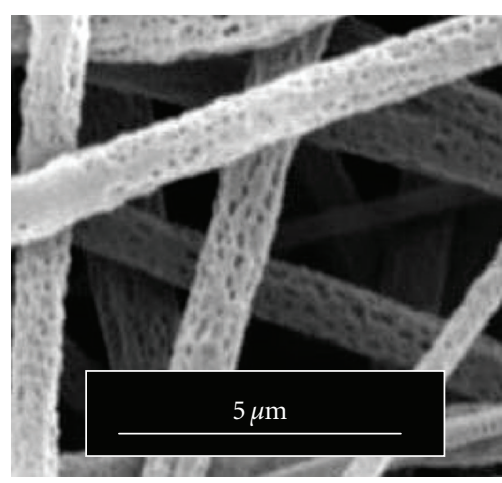

(b)

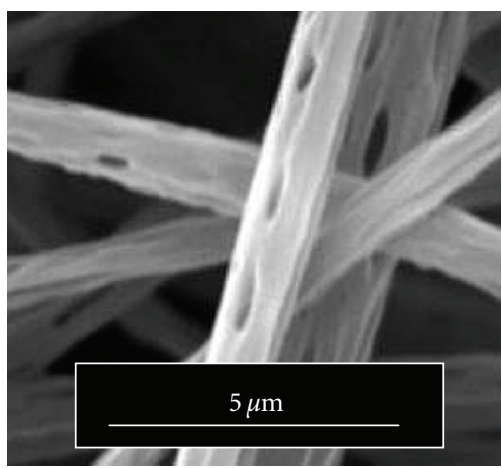

(d)

Figure 6: SEM images of PVB nanofibers prepared from (a) $10 \mathrm{wt} . \%$ THF/DMSO (9/1 v/v), (b) 8 wt.\% THF DMSO (95/5 v/v), (c) 8 wt.\% THF/DMSO ( $9 / 1 \mathrm{v} / \mathrm{v})$, and (d) $8 \mathrm{wt} . \%(8 / 2 \mathrm{v} / \mathrm{v})$.

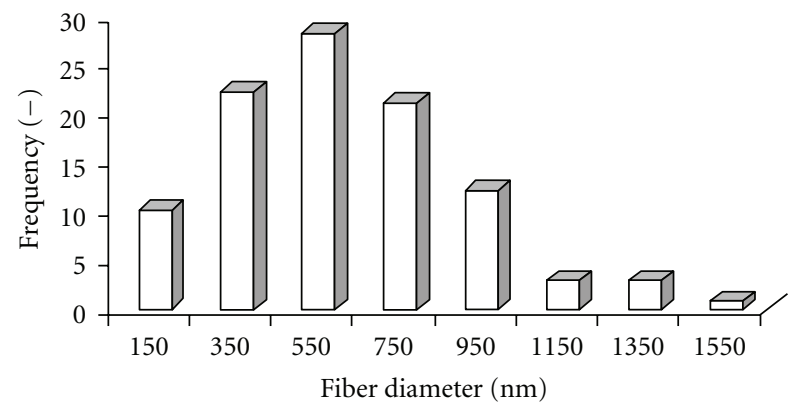

Figure 7: Histogram of the fiber diameter for electrospun PVB $8 \%$ wt. solution by the electrode-to-collector distance of $10 \mathrm{~cm}$ and applied voltage of $30 \mathrm{kV}$.

the creation of the porous nanofibers structure must be valid: (i) application of the mixture of good and poor solvents for the spinning solution, (ii) a distinct difference in the evaporation rate between good and poor solvents, and (iii) correct ratios of good and poor solvents $(\mathrm{v} / \mathrm{v})$. The abovementioned conditions were fulfilled for the preparation of porous and nonporous PVB fibers.

In the case of the electrospinning of PVB solutions from ethanol/methanol mixtures, smooth fibers were formed regardless of the composition of solvent mixture. Both solvents act as a poor solvent for ethanol, thus the first

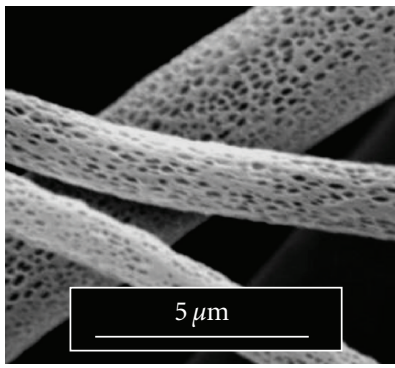

(a)

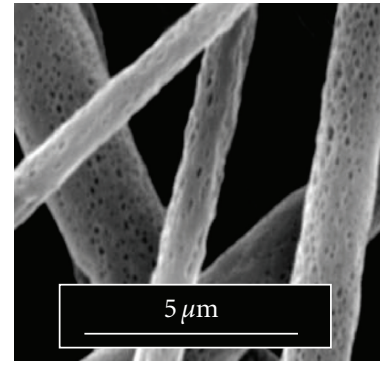

(b)
Figure 8: SEM images of PVB nanofibers prepared from $10 \mathrm{wt} . \%$ THF/DMSO (9/1 v/v): (a) applied voltage of $30 \mathrm{kV}$ (b) applied voltage of $35 \mathrm{kV}$ and an electrode-to-collector distance of $10 \mathrm{~cm}$.

condition is not fulfilled. In the case of the ethanol/DMSO $(9 / 1 \mathrm{v} / \mathrm{v})$ mixture, the indication of wrinkled fiber structure was evident. In this case the second condition (the difference in vapor pressure) was fulfilled. Nonporous fibers were formed in the case because both the solvents belong to the group of poor solvents for PVB. The first successful attempts to prepare porous nanofibers were realized by the spinning of the THF/DMSO ( $9 / 1 \mathrm{v} / \mathrm{v})$ mixture. This composition of the spinning solution fulfils all three basic conditions (mixture of the good and poor solvents, difference in the vapor pressure of individual solvents, and correct ratio of mixed solvents). 


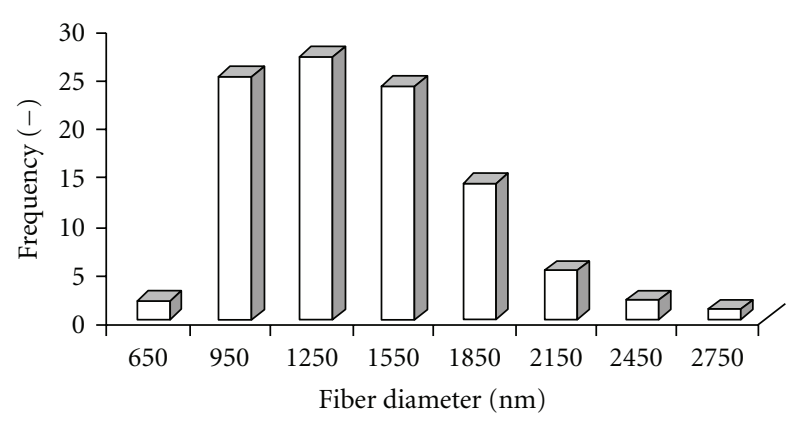

Figure 9: Histogram of the fiber diameter for electrospun PVB $10 \mathrm{wt} . \%$ solution from the electrode-to-collector distance of $10 \mathrm{~cm}$ and applied voltage of $35 \mathrm{kV}$.

Three qualities of PVB nanofibers were obtained: (i) nonporous, (ii) partially porous, and (iii) porous. The mixture of good and poor solvents applied for preparation of the polymer solution leads to porous or partially porous nanofibers through electrospinning. A qualitative and quantitative difference in porosity of the nanofibers is given by the different value of the evaporation rate of good and poor solvents. The correct ratios of the good and poor solvents have to be exactly defined. In the case of the ratio $9 / 1$ $(\mathrm{v} / \mathrm{v})$, the nanofibers were more porous than in the case of the ratio $8 / 2(\mathrm{v} / \mathrm{v})$. The smooth and finer nanofibers were obtained from the PVB solution in an ethanol/methanol mixture. Both these solvents are defined as poor for PVB. Experimental results showed that the decrease of the polymer concentration reduced the fiber diameter while the porous structure remained unchanged. In contrast, an increase in the applied voltage reduces the fiber diameter but porosity of the fibers is diminished.

It is expected that the porous fibers could find a utilization in filtration applications, in which the effective surface area of porous fibers is the advantage.

\section{Acknowledgments}

This work was supported by Grant no. 1M0554 and GAČR no. 304/07/1129.

\section{References}

[1] C. H. Kim, Y. H. Jung, H. Y. Kim, D. R. Lee, N. Dharmaraj, and K. E. Choi, "Effect of collector temperature on the porous structure of electrospun fibers," Macromolecular Research, vol. 14, no. 1, pp. 59-65, 2006.

[2] Y. You, J. H. Youk, S. W. Lee, B. M. Min, S. J. Lee, and W. H. Park, "Preparation of porous ultrafine PGA fibers via selective dissolution of electrospun PGA/PLA blend fibers," Materials Letters, vol. 60, no. 6, pp. 757-760, 2006.

[3] S. O. Han, W. K. Son, D. Cho, J. H. Youk, and W. H. Park, "Preparation of porous ultra-fine fibres via selective thermal degradation of electrospun polyetherimide/poly(3hydroxybutyrate-co-3-hydroxyvalerate) fibres," Polymer Degradation and Stability, vol. 86, no. 2, pp. 257-262, 2004.

[4] W. J. Elford, "Principles governing the preparation of membranes having graded porosities. The properties of "gradocol" membranes as ultrafilters," Transactions of the Faraday Society, vol. 33, pp. 1094-1104, 1937.

[5] J. D. Ferry, "Ultrafilter membranes and ultrafiltration," Chemical Reviews, vol. 18, no. 3, pp. 373-455, 1936.

[6] D. Lubasova and L. Martinova, "Formation mechanism of porous polycaprolactone nanofibers prepared by electrospinning," in Proceedings of the 42nd IUPAC Congress, Glasgow, Scotland, August 2009.

[7] Z. Qi, H. Yu, Y. Chen, and M. Zhu, "Highly porous fibers prepared by electrospinning a ternary system of nonsolvent/solvent/poly(l-lactic acid)," Materials Letters, vol. 63, no. 3-4, pp. 415-418, 2009.

[8] M. S. Khil, S. R. Bhattarai, H. Y. Kim, S. Z. Kim, and K. H. Lee, "Novel fabricated matrix via electrospinning for tissue engineering," Journal of Biomedical Materials Research Part B, vol. 72, no. 1, pp. 117-124, 2005.

[9] Ch. Hansen, Hansen Solubility Parameters: A User's Handbook, CRC Press/Taylor and Francis, New York, NY, USA, 2007.

[10] D. W. Van Krevelen, Properties of Polymers: Their Correlation with Chemical Structure; Their Numerical Estimation and Prediction from Additive Group Contributions, Elsevier, Amsterdam, The Netherlands, 1990. 

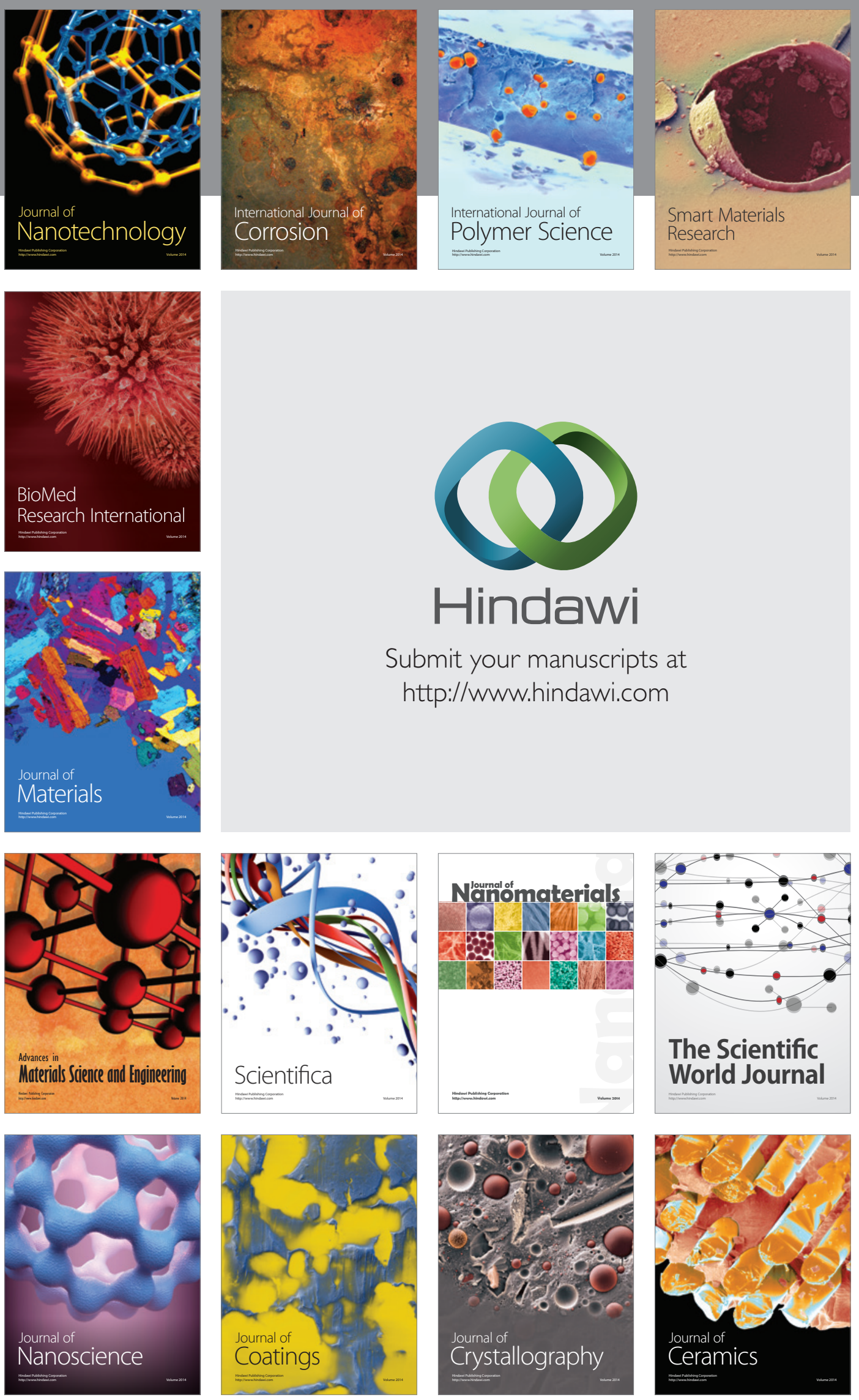

The Scientific World Journal

Submit your manuscripts at

http://www.hindawi.com

\section{World Journal}

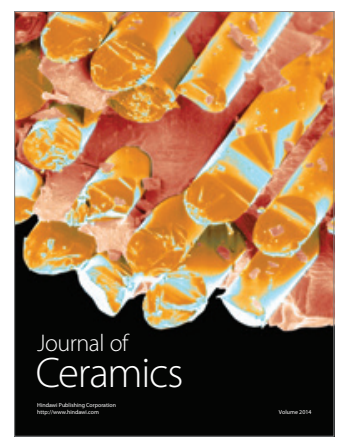

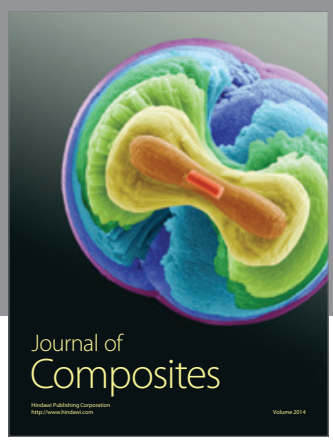
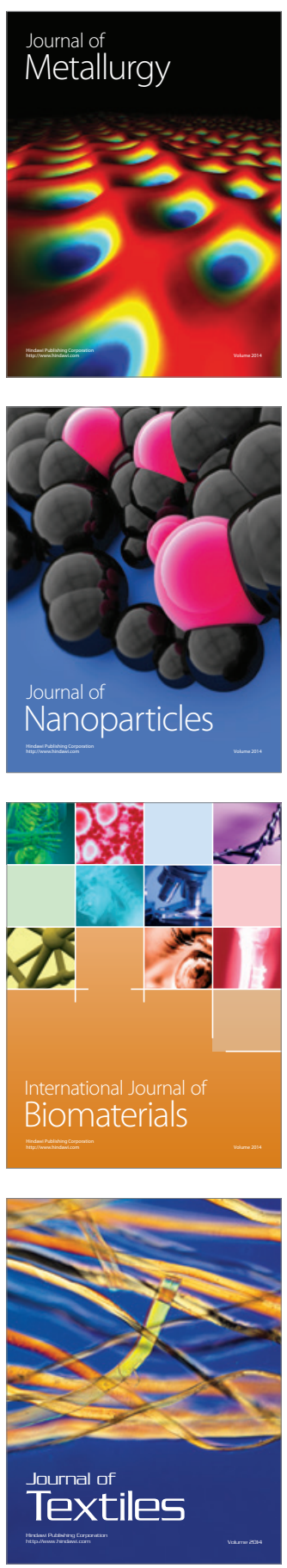